\title{
BMJ Open Systematic review protocol examining sex differences in survival among low birthweight newborns and infants in sub-Saharan Africa
}

Akalewold T Gebremeskel, ${ }^{1}$ Arone W Fantaye, ${ }^{2}$ Lena E Faust, ${ }^{3}$ Sanni Yaya (i) ${ }^{1,4}$

To cite: Gebremeskel AT, Fantaye AW, Faust LE, et al. Systematic review protocol examining sex differences in survival among low birthweight newborns and infants in subSaharan Africa. BMJ Open 2020;10:e036645. doi:10.1136/ bmjopen-2019-036645

- Prepublication history and additional material for this paper are available online. To view these files, please visit the journal online (http://dx.doi. org/10.1136/bmjopen-2019036645).

Received 23 December 2019 Revised 09 March 2020 Accepted 19 May 2020

Check for updates

(C) Author(s) (or their employer(s)) 2020. Re-use permitted under CC BY-NC. No commercial re-use. See rights and permissions. Published by BMJ.

${ }^{1}$ School of International Development and Global Studies, Faculty of Social Sciences, University of Ottawa, Ottawa, Ontario, Canada ${ }^{2}$ Interdisciplinary School of Health Sciences, Faculty of Health Sciences, University of Ottawa, Ottawa, Ontario, Canada ${ }^{3}$ Department of Epidemiology, Biostatistics and Occupational Health, Faculty of Medicine, McGill University, Montreal, Ontario, Canada

${ }^{4}$ The George Institute for Global Health, University of Oxford, Oxford, UK

Correspondence to

Dr Sanni Yaya;

sanni.yaya@u0ttawa.ca

\section{ABSTRACT}

Introduction In sub-Saharan African countries, low birthweight (LBW) accounts for three-quarters of under-five mortality and morbidity. However, there is no systematic evidence of sex differences in LBW survival risk. The aim of this protocol is to outline the methodological process of a systematic review that will gather qualitative and quantitative data on sex differences in survival among LBW newborns and infants in subSaharan Africa.

Methods This protocol adheres to the Preferred Reporting Items for Systematic Review and Meta-Analysis Protocols reporting guidelines. We will conduct a systematic review to retrieve all qualitative and quantitative studies. Electronic search strategies are being finalised on 24 February 2020 for Ovid Medline and EMBASE, and on 28 February 2020 for CINAHL, Scopus and Global Health in collaboration with a Health Sciences librarian. The primary outcome of interest is indicating sex differences in survival among LBW newborns and infants. Secondary outcomes are sex-disaggregated differences in morbidity among LBW newborns and infants. Screening, data extraction and assessments of risk of bias will be performed independently. Narrative synthesis and a meta-analysis will be conducted with studies that are compatible based on population and outcome. The systematic review is focused on the analysis of secondary data and does not require ethics approval.

Ethics and dissemination As it will be a systematic review, without human participants' involvement, there will be no requirement for ethical approval. The systematic review will present key evidence of sex-disaggregated differences in mortality and morbidity among LBW newborns and infants in sub-Saharan Africa. Programme managers, policy-makers and researchers can use the findings to evaluate LBW health outcomes in different sexes. The final manuscript will be disseminated through a peer-reviewed journal and scientific conferences. PROSPERO registration number CRD42020163470

\section{INTRODUCTION}

In 2015, the prevalence of low birthweight (LBW) was approximately $14.6 \%$, amounting to 20.5 million LBW babies. ${ }^{1}$ Approximately $91 \%$ of these LBW live births were from low-income and middle-income countries. ${ }^{2}$
Strengths and limitations of this study

- This protocol outlines the methodological process of a systematic review that will gather qualitative and quantitative data on sex differences in survival among low birthweight (LBW) newborns and infants in sub-Saharan Africa (SSA). Also, this reduces the possibility of duplication and possible biases.

- This protocol is developed to offer the highest level of evidence for informed decisions by organising an experienced multidisciplinary team.

- This systematic review protocol will be the first to present key evidence of sex-disaggregated differences in mortality (survival) and morbidity among LBW newborns and infants in SSA.

- Significant numbers of LBW data are unrecorded, under-reported and misreported by birthweight and sex, possibly resulting in publication bias and methodological quality issues.

- Exclusion of studies not disaggregating survival outcomes in LBW newborns and infants by sex as well as exclusion of research published before 2000, in non-SSA regions and in languages other than English.

Accordingly, the WHO identified LBW as an indicator of child health status. ${ }^{3}$ Birth weight of less than $2500 \mathrm{~g}$ is classed as LBW, regardless of gestational age. ${ }^{4}$

It is also a major determinant of infant mortality, morbidity, and poor mental and physical development. $^{5}$ The neonatal and infant periods are vulnerable periods for child survival. ${ }^{6} 7$ LBW accounts for approximately $80 \%$ of all newborn deaths. ${ }^{2}$ Although LBW is among the strongest predictors of infant morbidity and mortality in most parts of the developing world, in Africa, it is the strongest predictor. $^{8}$ Sub-Saharan Africa (SSA), where approximately $15 \%$ of neonates are born with LBW, ${ }^{2}$ accounts for a quarter of the global burden of LBW live births. ${ }^{29}$ This region also constitutes the highest neonatal and under-five mortality rates in the world. ${ }^{67}$ 
LBW-related mortality continues to be a significant global and public health challenge. Ensuring universal health access is unthinkable without children, who make up a large and relatively dependent part of the population. In 2015, Sustainable Development Goal (SDG) 3, target 3.2 aimed to reduce newborn mortality to 12 per 1000 live births by $2030 .^{10}$ It also set a target to reduce under-five mortality to 25 per 1000 live births, and LBWs to $30 \%$ by $2030 .^{10}$

Available research evidence has identified various risk factors for mortality among LBW newborns and infants. ${ }^{11-14}$ One of the more contested individual-level risk factors is sex, which is a key variable for disaggregation of child mortality and morbidity rate estimates. Organising sex-disaggregated data is an important component of gender analysis, in which quantifiable differences are made between male and female individuals. ${ }^{15}$ Most UN health indicators are sex disaggregated ${ }^{16}{ }^{17}$ : 'Sexdisaggregated data allow programme managers and decision makers to evaluate service quality, treatment, and health-outcome in different sexes'. ${ }^{18}$

Despite the significant influence of LBW on adverse health outcomes, there is a lack of evidence synthesis on this key public health concern across SSA. Therefore, this systematic review primarily evaluates sex differences in survival among LBW newborns and infants in SSA. It synthesises the existing evidence on sex-disaggregated differences in survival and morbidity outcomes in this population. The availability of sex-disaggregated LBW and mortality data can be crucial in informing interventions aiming for SDG 3.2 targets. In addition to identifying the existing evidence, the review will identify evidence gaps in the literature for sex-specific LBW outcomes. Review findings will ultimately inform programme implementers, policy-makers and researchers addressing LBW-related mortality and morbidity. To our knowledge, there is no existing systematic review on this research aim in SSA.

\section{Objective}

The aim of this protocol is to outline the methodological process of a systematic review that will gather qualitative and quantitative data on sex differences in survival and morbidity among LBW newborns and infants in SSA.

\section{Research question}

Are there sex differences in LBW mortality and morbidity outcomes among newborns and infants in SSA?

\section{METHODS}

\section{Study design}

This protocol was designed and written according to the Preferred Reporting Items for Systematic Review and Meta-analysis Protocols guideline for reporting systematic reviews (see the online supplementary file 1). The protocol has been registered in PROSPERO, an international prospective register of systematic reviews.

\section{Data sources and search strategy for relevant studies}

Electronic search strategies are being finalised for Ovid Medline and EMBASE on 24 February 2020, and CINAHL, Scopus and Global Health on 28 February 2020. This was done in collaboration with a Health Sciences librarian, who helped in optimising the retrieval of relevant citations. Search strategies include variations, mesh terms, and explore or narrowed versions of the following keywords: LBW, preterm, premature, small for gestational age (SGA), newborn, infant, sex, male, female and SSA (see the online supplementary file 2).

The search strategies designed to access published materials comprise three stages. (1) A limited search of Ovid Medline and CINAHL to identify relevant keywords contained in the title, abstract and subject descriptors. (2) Terms identified in this way, and the synonyms used by Ovid Medline, EMBASE, CINAHL, Global Health and Scopus are used in an extensive search of the literature. (3) Reference lists of the review eligible full-text articles will be perused to identify more relevant articles.

\section{Eligibility criteria}

We have proposed a clear and predefined eligibility criterion for this systematic review (see the online supplementary file 3 ).

Inclusion criteria: peer-reviewed full-text research articles, published in English, will be considered. Articles published between October 2000 and 2019 will be considered. This period was selected because the years 2000 to 2015 represent the era of Millennium Development Goals, where significant progress was made around the world, including in infants' health. As a continuation of the increased focus of research on child health, the Sustainable Development Goals adopted in 2015 set new targets to reduce child mortality and improve child health by 2030 . The period from 2000 to 2019 thereby accounts for the new wave of research related to development goals on reducing child mortality, since the turn of the century. Quantitative, qualitative and mixed-method studies conducted in SSA will be included. The review will also consider all types of studies on male and female LBW newborns and infants with birth weights lower than $2500 \mathrm{~g}$ in SSA. Studies that report sex differences in survival or morbidity among LBW newborns or LBW infants at the time of discharge from a health facility will be included.

Exclusion criteria: studies conducted outside of SSA countries and studies that do not report sex differences or solely report sex differences in a population of newborns and infants will be excluded. Studies that only include preterm and SGA babies that are not LBW (weighing over $2500 \mathrm{~g}$ ) will be excluded. Preterm and SGA are not synonyms of LBW, and in consort, preterm and SGA babies can be normal weight. Given that the review explores sex differences in survival, studies that do not report the weight of the population or disaggregate the sexes in LBW survival or morbidity outcomes will also be excluded. Lastly, all non-primary literature sources, such as systematic reviews, theses and dissertations, will 
be excluded to ensure the focus of the review on peerreviewed, full-text academic articles.

\section{Population}

LBW male and female newborns born in SSA ( $<28$ days of age).

LBW male and female infants born in SSA $(<1$ year of age).

\section{Intervention(s), exposure(s)}

There is no specific intervention targeted for this study.

\section{Comparison}

The usual standard of care without intervention.

\section{Outcomes}

The primary outcome of interest is sex differences in the survival (mortality) of LBW newborns and infants at the time of discharge from a health facility.

The secondary outcomes of interest include sex differences in morbidity, such as non-communicable and communicable diseases, of LBW newborns and infants at the time of discharge from a health facility.

\section{Screening}

Citations will be imported into the Mendeley citation management software and duplicates will be removed. The articles retrieved from searches in the databases will be screened by three authors in the Rayyan database for their relevance and eligibility to be included in the review. This will include the title and abstract screening, followed by full-text screening against the eligibility criteria for studies deemed potentially eligible. Disagreements will be settled through discussion.

\section{Data extraction}

After full-text screening, data will be independently extracted from the retrieved eligible studies by two of the reviewers (ATG and AWF). Disagreements will be settled through discussion with a third reviewer (SY). The authors will adapt a data collection form based on the needs of the review from a standardised data extraction form by the Cochrane library. ${ }^{19}$ The data extracted will include all details specific to the review question, fulfilling the requirements for both the narrative synthesis and the potential meta-analysis. This includes the following information from each article: (1) authors and publication year, study setting, and study aim or hypothesis; (2) sample characteristics, design and data collection methods, outcome measures, statistical analyses; (3) study findings. We will also contact primary study authors for key information when data are ambiguous or missing from the included studies.

\section{Data synthesis}

A narrative synthesis will be conducted, a method that is ideal for synthesising evidence from a wide range of research questions and study designs with quantitative and qualitative approaches. ${ }^{20}$ If the data are too heterogeneous as anticipated, the narrative synthesis will be solely conducted. Descriptive statistics will be provided on all included studies, in a way that indicates regional study results (East, West, South and Central Africa). Data on study characteristics, outcomes and important variables will be summarised using frequencies and percentages for dichotomous outcomes. Where sufficient data are available, a meta-analysis will be conducted, in $\mathrm{R}$ v.3.6.2. Due to heterogeneity in geographic and sociodemographic factors across studies, a random-effects model will be used for the meta-analysis. The specific method employed to produce pooled estimates will depend on the study designs and analyses of the included studies. However, as anticipated, the included studies will present time-to-event data (ie, survival analyses). The inverse variance method (conducted using the R package 'meta') will be used to pool study estimates, based on the reported HRs and corresponding SEs. Statistical heterogeneity will be assessed via the Higgin's I ${ }^{2}$ statistic.

\section{Risk of bias assessment for retained studies}

The risk of bias in included studies will be assessed independently by two reviewers, with discrepancies resolved by the corresponding author. The reviewers will evaluate the qualitative and quantitative studies using the appropriate Critical Appraisal Skills Programme (CASP) checklists. ${ }^{21}$ The domains of the CASP checklists will help to assess the credibility of the findings and the rigour of the studies. ${ }^{22}$ The questions were designed as prompts to guide reviewers in critically reading the reports. Included studies will be assigned an overall score of 'high' (9-10), 'moderate' (7.5-9) or 'low' (less than 7.5) overall quality. Studies will not be excluded or weighted based on the quality of the reporting assessment. The results of the appraisal will instead be used to inform data interpretation and help confirm the validity of review findings and conclusions. In a quantitative meta-analysis, study heterogeneity and publication bias will consider extended funnel plot tests for detecting publication bias, and selection modelling and trim-and-fill methods to adjust for publication bias in the presence of between-study heterogeneity.

\section{Quality of review evidence assessment}

We will use the Grading of Recommendations, Assessment, Development and Evaluations (GRADE) framework to assess the strength of evidence for each outcome from included studies. ${ }^{23}$ The quality of each piece of evidence for primary and secondary outcomes will be classified as 'high', 'moderate', 'low' or 'very low'.

\section{Patient and public involvement}

Patients were not directly involved in the design of this study. As this is a protocol for a systematic review and no participant recruitment will take place, their involvement in the recruitment and dissemination of findings to participants was not applicable. 


\section{Ethics and potential amendments}

Ethics approval is not required as the systematic review does not involve the collection of primary data from participants. The collection of data for our review does not involve direct contact with human participants. Instead, we will use published and publicly accessed data. We do not envisage any amendments to the present protocol, but should an amendment be necessary, it will be notified, registered and reported.

\section{Dissemination of findings}

The systematic review and its evidence synthesis will be published in a peer-reviewed journal and presented at different conferences and scientific meetings. The findings will be used to inform the design of sex-specific interventions aiming to improve outcomes of LBW newborns and infants across SSA in the future. The findings will also be used to identify gaps in the literature evidence regarding mortality and morbidity among LBW newborns and infants.

\section{Twitter Sanni Yaya @realSanniYaya}

Contributors SY led the design and coordination of the review. ATG and AWF developed the search strategies in collaboration with a librarian. LEF planned the statistical analysis and provided critical insights. ATG, AWF and LEF will conduct the screening of the articles, extract the data, appraise the quality of evidence, analyse the data and write the report. SY had final responsibility to submit for publication. All authors were responsible for revising the protocol manuscript critically for important intellectual content. All authors read and approved this final protocol manuscript.

Funding The authors have not declared a specific grant for this research from any funding agency in the public, commercial or not-for-profit sectors.

Competing interests None declared.

Patient and public involvement Patients and/or the public were not involved in the design, or conduct, or reporting, or dissemination plans of this research.

Patient consent for publication Not required.

Provenance and peer review Not commissioned; externally peer reviewed.

Open access This is an open access article distributed in accordance with the Creative Commons Attribution Non Commercial (CC BY-NC 4.0) license, which permits others to distribute, remix, adapt, build upon this work non-commercially, and license their derivative works on different terms, provided the original work is properly cited, appropriate credit is given, any changes made indicated, and the use is non-commercial. See: http://creativecommons.org/licenses/by-nc/4.0/.

\section{ORCID iD}

Sanni Yaya http://orcid.org/0000-0002-4876-6043

\section{REFERENCES}

1 The United Nations Children's Fund,, World Health Organization, The London School of Hygiene \& Tropical Medicine,, Johns Hopkins University. Low birthweight estimates: levels and trends 2000-2015. New York: UNICEF, 2019. https://www.unicef.org/media/53711/file/ UNICEF-WHO\%20Low\%20birthweight\%20estimates\%202019\%20. pdf
2 Blencowe $\mathrm{H}$, Krasevec J, de Onis M, et al. National, regional, and worldwide estimates of low birthweight in 2015, with trends from 2000: a systematic analysis. Lancet Glob Health 2019;7:e849-60.

3 World Health Organization. The world health report 1998. life in the 21st century. A vision for all. Geneva: World Health Organization, 1998. https://www.who.int/whr/1998/en/whr98_en.pdf?ua=1

4 Kramer MS. Determinants of low birth weight: methodological assessment and meta-analysis. Bull World Health Organ 1987;65:663-737.

5 Roudbari M, Yaghmaei M, Soheili M. Prevalence and risk factors of low-birth-weight infants in Zahedan, Islamic Republic of Iran. East Mediterr Health J 2007;13:838-45.

6 United Nations Children's Fund. Neonatal mortality [Internet]. UNICEF DATA, 2019. Available: https://data.unicef.org/topic/childsurvival/neonatal-mortality/ [Accessed 2 Sep 2019].

7 United Nations Children's Fund. Under-five mortality [Internet]. UNICEF DATA., 2019. Available: https://data.unicef.org/topic/childsurvival/under-five-mortality/ [Accessed 2 Sep 2019].

8 Elshibly EM, Schmalisch G. The effect of maternal anthropometric characteristics and social factors on gestational age and birth weight in Sudanese newborn infants. BMC Public Health 2008;8:244.

9 United Nations Children's fund. Low birthweight [Internet]. UNICEF DATA, 2019. Available: https://data.unicef.org/topic/nutrition/lowbirthweight/ [Accessed on 12 September 2019].

10 United Nations. Sustainable development goal 3, N.d. Available: https://sustainabledevelopment.un.org/sdg3 [Accessed 7 Sep 2019].

11 Bacak SJ, Baptiste-Roberts K, Amon E, et al. Risk factors for neonatal mortality among extremely-low-birth-weight infants. Am J Obstet Gynecol 2005;192:862-7.

12 Nayeri F, Dalili H, Nili F, et al. Risk factors for neonatal mortality among very low birth weight neonates. Acta Med Iran 2013;51:297-302.

13 Gaiva MAM, Fujimori E, Sato APS. [Neonatal mortality in infants with low birth weight]. Rev Esc Enferm USP 2014;48:778-86.

14 Castro ECMde, Leite Álvaro Jorge Madeiro, Almeida MFBde, et al. Perinatal factors associated with early neonatal deaths in very low birth weight preterm infants in northeast Brazil. BMC Pediatr 2014;14:312.

15 Assist U. Gender Considerations in Monitoring and Evaluation [Internet]. USAID ASSIST PROJECT, n.d. Available: https://www. usaidassist.org/resources/gender-considerations-monitoring-andevaluation [Accessed 2 Sep 2019].

16 United Nations. Progress on the sustainable development goals. The gender snapshot 2019. New York: United Nations, 2019. https:// unstats.un.org/sdgs/report/2019/gender-snapshot.pdf

17 Sawyer CC. Child mortality estimation: estimating sex differences in childhood mortality since the 1970s. PLoS Med 2012;9:e1001287.

18 Evaluation M. Barriers to and Facilitators of Sex and AgeDisaggregated Data - Kenya. Chapel Hill: MEASURE Evaluation, 2017. https://www.measureevaluation.org/resources/publications/ tr-17-163

19 Cochrane. EPOC resources for review authors [Internet]. EPOC resources for review authors |Cochrane Effective Practice and Organization of Care. Cochrane. Available: https://epoc.cochrane. org/resources/epoc-resources-review-authors [Accessed 3 Oct 2019].

20 Popay J, Roberts H, Sowden A, et al. Guidance on the conduct of narrative synthesis in systematic reviews: a product from the ESRC methods programme, version 1. Lancaster: Institute of Health Research, 2006. http://citeseerx.ist.psu.edu/viewdoc/download?doi= 10.1.1.178.3100\&rep=rep1\&type=pdf

21 Critical Appraisal Skills Programme. CASP Checklists - Critical Appraisal Skills Programme [Internet]. CASP Appraisal Checklists. CASP, 2018. Available: https://casp-uk.net/casp-tools-checklists/ [Accessed 10 Feb 2020].

22 Singh J. Critical appraisal skills programme. J Pharmacol Pharmacother 2013;4:76-7.

23 Lasserson T, Santesso N, Cumpston M, et al. Incorporating grade in Cochrane reviews. Cochrane editorial unit, 2014. Available: https:// training.cochrane.org/resource/incorporating-grade-cochranereviews [Accessed 14 Oct 2019]. 